\title{
In Vivo Study of Natural Killer (NK) Cell Cytotoxicity Against Cholangiocarcinoma in a Nude Mouse Model
}

\author{
IN HYE JUNG ${ }^{1 *}$, DO HEE KIM ${ }^{1,2^{*}}$, DA KYUNG YOO $^{3}$, SUN YOUNG BAEK ${ }^{3}$, SEONG HOON JEONG ${ }^{3}$, \\ DAWOON E. JUNG ${ }^{1}$, SEUNG WOO PARK ${ }^{1}$ and YONG-YOON CHUNG ${ }^{3}$ \\ ${ }^{1}$ Institute of Gastroenterology, Yonsei University College of Medicine, Seoul, Republic of Korea; \\ ${ }^{2}$ Postgraduate School of Nano Science and Technology, Yonsei University, Seoul, Republic of Korea; \\ ${ }^{3}$ Research Institute of SMT Bio, SMT Bio Co., Ltd., Seoul, Republic of Korea
}

\begin{abstract}
Background/Aim: Natural killer (NK) cells are one of the lymphocytes clinically used for various cancer types. Cytotoxicity of $\mathrm{NK}$ cells to cholangiocarcinoma $(\mathrm{CC})$, however, has not yet been studied. Nor NK cell therapy against CC has been clinically applied. In this study, relevance of $\mathrm{NK}$ cell therapy for anti-tumor efficacy against CC was pre-clinically investigated. Materials and Methods: Human HuCCT-1 cells, an intrahepatic CC cell line, were xenografted into nude mice. The HuCCT-1 tumor-bearing nude mice then received multiple infusions of ex vivoexpanded human NK cells (SMTO1) and in vivo cytotoxic activity of the NK cells against the CC cells was evaluated. Results: SMT01 infusion resulted in significant inhibition of the CC tumor growth. Body weight of the mice administrated with chemotherapy was found to be maintained at the lowest level among all treatment groups while all the SMTO1 infusion groups well maintained their body weight. Conclusion: The present in vivo study demonstrates that NK cells contain cytolytic activity against cholangiocarcinoma and show beneficial effect of $N K$ cell therapy in relevance to quality of life. Further investigation of the NK cell-based immunotherapy can be useful to determine cancer therapeutics for the specific tumor.
\end{abstract}

This article is freely accessible online.

*These Authors contributed equally to this study.

Correspondence to: Yong Yoon Chung, Research Institute of SMT Bio, SMT Bio Co., Ltd. Non-hyun Ro, 114 Gil 5, Kangnam Gu, Seoul, 06108, Republic of Korea. Tel: +82 234610515, e-mail: yjung21@gmail.com; Seung Woo Park, Yonsei-Ro 50, SeodaemunGu, Seoul, Republic of Korea, 120-749. Tel: +82 1038021965, email: swoopark@yuhs.ac

Key Words: Cholangiocarcinoma, NK cell, xenograft nude mouse model.
Cholangiocarcinoma (CC) is a type of cancer that arises from the epithelium of bile duct. $\mathrm{CC}$ is a relatively rare cancer with an annual incidence of $<6$ cases per 100,000 persons worldwide, but the incidence number keeps increasing, especially in certain Asian countries including China, South Korea, and Thailand (1-3). CC is classified as an invasive adenocarcinoma which is considered to be incurable, when diagnosed at an advance stage. The 5-year survival rate for people with early-stage extrahepatic and intrahepatic CC is $30 \%$ and $15 \%$, respectively. However, most patients are found to be inoperable at advanced stages of the disease at the time of diagnosis which results in 2-5\% 5-year survival rate. While no potentially curative treatment for $\mathrm{CC}$ exists, gemcitabine/cisdiammineplatinum (II) dichloride (Gem/CDDP) regimen is widely administered to patients at an inoperable stage.

Natural killer (NK) cells are one of the lymphocytes with natural cytotoxicity, recognizing and lysing malignant and virally infected cells without prior sensitization $(4,5)$. These cells are now widely used as an innovative immune-therapy against various types of cancers lacking major histocompatibility complex (MHC) class I, while normal cells expressing MHC I molecules are recognized by the NK cell's receptor, killer cell immunoglobulin-like receptor (KIR), inhibiting the NK cell's cytotoxicity $(6,7)$. The NK activating signaling is conferred by NKG2D (natural-killer group 2D), a lectin-like activating receptor $(8,9)$. When MHC I expression is lost or down-regulated in cancer cells, the NK inhibitory signal is abrogated and NK cells are activated to kill the malignant target cancer cells. Identification of NK cells can be done by detecting CD56 or CD16 expression and the absence of CD3 expression (10, 11). The CD56 expressing NK cells can be further divided into two subsets, CD56 ${ }^{\text {bright }}$ and CD56 ${ }^{\text {dim }} \mathrm{NK}$ cell population (12).

Clinical use of NK cells against various cancers has already been performed. The first trials in humans focused on the use of autologous NK cells. The efficacy, however, was limited due to autologous NK cell inhibition by self- 
MHC. As the NK cell receptors were further characterized, the next clinical approach using NK cells focused on allogeneic NK cells obtained from healthy haploidentical donors. This approach has allowed NK cells to avoid tumorinduced suppression and demonstrated greater tumor-killing activity $(13,14)$. Indeed, the strategy of administrating allogeneic NK cells to cancer patients has adoptively been used (15).

No immune cell therapy including NK cells for CC has been yet developed. There are no research reports on the usage of the NK cells for the treatment $\mathrm{CC}$. The primary goal of this study was to investigate whether NK cell therapy has any clinical anti-tumor efficacy against $\mathrm{CC}$. To this direction, we examined whether NK cells are cytotoxic to CC cells and can be used as cancer therapeutics.

\section{Materials and Methods}

Study design of the in vivo study in Nude mice. $\mathrm{NCr}$ athymic nude mice (BALB/cSlc-nu/nu) (Charles River Laboratories, Atsugi, Japan SLC, Inc) were handled and housed in microisolator cages following OECD Good Laboratory Practice regulations (ENV/MC/CHEM(98)17 as revised in 1997) and Good Laboratory Practice for Nonclinical Laboratory Studies (21CFR Part 58, Food and Drug Administration, United States of America (Apr. 1, 2015). To evaluate dosage dependent safety and toxicity, three different doses of SMT01 (NK cells) were determined (Figure 1A): Low dose infusion of $4 \times 10^{4}$ cells/animal which corresponds to $2 \times 10^{6}$ cells $/ \mathrm{kg}$ in human. Intermediate and high doses of $2 \times 10^{5}$ cells/animal and $1 \times 10^{6}$ cells/animal, respectively. The ex vivo expanded NK cells were then intravenously injected (tail vein injection at $2 \mathrm{ml} / \mathrm{min}$ using $26 \mathrm{G}$ syringe, $0.2 \mathrm{ml} /$ animal) to 50 male and female mice (weight range 18.7 22.5 $\mathrm{g}$ and $16.1 \sim 18.8 \mathrm{~g}$, respectively, at 6 weeks) at 2 times per 3 weeks for 27 weeks. A total of 18 SMT01 infusions were performed. The same nude mice were used for the dosage efficacy study (Figure 1B). To do this, transplantation and engraftment was firstly done by subcutaneous injection of HuCCT1 cells $\left(5 \times 10^{6}\right.$ cells $\left./ 0.2 \mathrm{ml}\right)$ into 10 nude mice per group (G1-G5): G1, normal saline (negative control); G2-G4, SMT01 infusions; G5, Gem+CDDP (positive control). Eight well engrafted nude mice with a $84 \sim 119 \mathrm{~mm}^{3}$ tumor volume (19.3 20.5 g body weight range) from each group were then selected and treated.

The nude mice bearing a HuCCT-1 tumor were administered intravenously with SMT01 5 times with 10 days of interval. Chemoadministration as a positive control group was also done with Gemcitabine (Gem) and Cis-diammineplatinum (II) dichloride (CDDP) at $120 \mathrm{mg} / \mathrm{kg}$ and $3 \mathrm{mg} / \mathrm{kg}$, respectively. SMT01 infusion was performed to three different mice groups (Table I): SMT01 infusions, G2-G4: G2, low dose $\left(4 \times 10^{4}\right.$ cell/animal); G3, intermediate dose $\left(2 \times 10^{5}\right.$ cells/animal $) ; \mathrm{G} 4$, high dose $\left(1 \times 10^{6}\right.$ cells/animal). G1 and G5, negative control (normal Saline) and positive control (CDDP+Gem), respectively. Cell injection was done by using a disposable syringe $(26 \mathrm{G}, 1 \mathrm{ml})$. Injection volume was $0.2 \mathrm{ml} / \mathrm{animal}$. At 14 days after the final infusion, the tumor-bearing mice were sacrificed and processed for evaluation (Figure 1B).

Preparation and ex vivo expansion of NK cells. Blood was obtained alternatively from two healthy donors and used for peripheral blood mononuclear cell (PBMC) isolation during the preclinical study. $\mathrm{CD}^{+} \mathrm{T}$ cell depletion was done by using MACSxpress (Milteyi Biotec., Seoul, Korea). The T cell depleted PBMC was washed two times with DPBS buffer and cultured in a T75 flask containing 20 $\mathrm{ml}$ of an NK expansion medium (ALyS505NK-IL2 1,000 IU/ml, Cell Science \& Technology Institute Inc., Sendai, Japan). The IL-2 activated NK cells were fed with fresh medium every three days and transferred to a T175 flask after 5-7 days of culture. The NK cell expansion was continued for another 7 to 14 days by adding fresh medium until a desired cell number was reached. The viability and number of the expanded NK cells was performed by the trypan blue counting method with an automatic cell counter.

Human cell lines. Human biliary tract cancer cell lines used for the study were: HuCCT-1 (intrahepatic) purchased from the Health Science Research Resources Bank (Osaka, Japan), and SNU1196 (extrahepatic), SNU308 (extrahepatic), and SNU478 (ampulla of Vater) obtained from the Korean Cell Line Bank (Seoul, Korea). The cell lines were cultured in RPMI-1640 medium (GIBCO, Seoul, Korea) supplemented with $10 \%$ fetal bovine serum (GIBCO), 100 $\mathrm{U} / \mathrm{ml}$ penicillin, and $100 \mathrm{mg} / \mathrm{ml}$ streptomycin in humidified atmosphere containing $5 \% \mathrm{CO}_{2}$.

Phenotypic flow cytometric analysis. To analyze cell populations of SMT01, the cells were stained with PE conjugated anti-human CD56 and anti-human CD40, and FITC conjugated anti-human CD3 and anti-human CD16 monoclonal antibodies (BD Biosciences, Seoul, Korea). For staining, SMT01 were washed twice in phosphate buffer saline (PBS) and re-suspended in FACS staining buffer (PBS with $0.5 \%$ bovine serum albumin). The cells were then stained with the appropriate antibodies at $4{ }^{\circ} \mathrm{C}$ for $30 \mathrm{~min}$. After washing with PBS buffer three times, the cells were analyzed on a FACS flowcytometer (Beckton Dickinson AriaIII). Gates were set around $\mathrm{NK}$ cells defined as $\mathrm{CD} 56^{+} / \mathrm{CD} 3^{-}$and $\mathrm{CD} 56^{+} / \mathrm{CD} 16^{+}$to determine NK cell population.

Cytotoxicity assay. Cytolytic NK cell activity was measured by using Cell Counting Kit-8 (CCK-8) (Dojindo Mol. Tech., Rockville, MD, USA). K562 cells were included as a positive target cell to compare cytolytic activity of the NK cell against human cholangiocarcinoma cell lines. SMT01 effector cells were seeded into the 96-well plates at a density of $1 \times 10^{4}$ cell per well and incubated for $24 \mathrm{~h}$. Cell viability of the target cell lines at three different effector:target (E:T) cell ratios $(1: 5,1: 1$, and 5:1) was measured by CCK 8 kit following the manufacturer's instructions. Absorbance was measured at $450 \mathrm{~nm}$ using a microplate reader. Cytotoxic effect was calculated as follows. Cytotoxicity $(\%)=100 \%$ - $((\mathrm{ODe}+\mathrm{t}-\mathrm{ODb})-(\mathrm{ODe}-\mathrm{ODb})) /(\mathrm{ODt}-\mathrm{ODb}) \times 100 \%(\mathrm{OD}$, absorbance; e, effector; $t$, target; $b$, blank).

Histology, immunohistochemistry (IHC) and TUNEL assay. For histologic evaluation, HuCCT-1 tumor was dissected out from the nude mice and embedded into paraffin. Four $\mu \mathrm{m}$ tissue sections were prepared and stained with hematoxylin and eosin (H\&E) according to standard protocols (16). The same tissue sections were used for IHC experiments. IHC experiments were carried out as previously described $(17,18)$. Antibodies used for IHC experiment were anti-human CD56 (BD Sciences, Seoul, Korea), natural cytotoxicity triggering receptor 1 (NCR1, BD Sciences), and antihuman Caspase 3a antibody (Abcam, Seoul, Korea). The tumor 


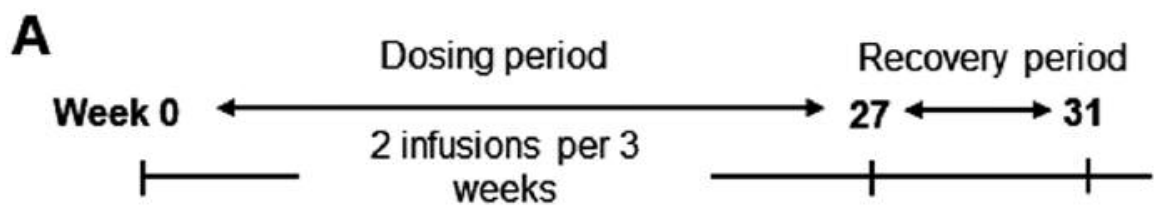

G1, 0 cells/animal; G2, $4 \times 10^{4}$ cells/animal; G3, $2 \times 10^{5}$ cells/animal; G4, $1 \times 10^{6}$ cells/animal

B

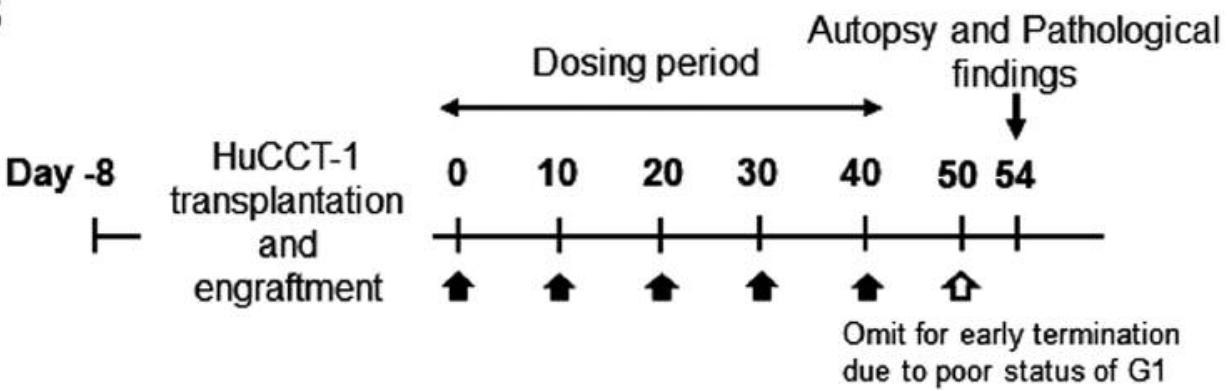

\section{G1, 0 cells/animal; G2, $4 \times 10^{4}$ cells/animal; G3, $2 \times 10^{5}$ cells/animal; G4, $1 \times 10^{6}$ cells/animal; G5, Gem $(120 \mathrm{mg} / \mathrm{kg})+\operatorname{CDDP}(3 \mathrm{mg} / \mathrm{kg})$. Arrowhead, injection treatment.}

Figure 1. Study design for the in vivo study in nude mice. A: Dosage-dependent safety and toxicity. B: Dosage efficacy study. The HuCCT-1 xenografted nude mice were initially planned to receive 6 treatments. Since the G1 group (no treatment group) however showed poor status with a significant tumor growth, 6th treatment was omitted.

Table I. Dose escalation study of SMT01.

Group (Number of animal) Injected cell number (cells/animal) Injected volume (mL/animal)

Mean proliferation rate of Lymphocyte (\%)

T cell (male/female) B cell (male/female)

\begin{tabular}{|c|c|c|c|c|c|}
\hline G1 & Negative control (20) & 0 & 0.2 & $100 / 100$ & $100 / 100$ \\
\hline G2 & Low dose (20) & $4 \times 10^{4}$ & 0.2 & $97.5 / 99.1$ & $99.8 / 102.2$ \\
\hline G3 & Intermediate dose (20) & $2 \times 10^{5}$ & 0.2 & $98.8 / 103.3$ & $104.2 / 102.2$ \\
\hline G4 & High dose (20) & $1 \times 10^{6}$ & 0.2 & $97 / 104.2$ & $104.2 / 97.6$ \\
\hline
\end{tabular}

Negative control: normal saline.

section slides were also used to conduct in situ detection of apoptotic cells by TUNEL staining. The TUNEL assay was done by using TumorTACS in situ Apoptosis kit (R\&D Systems, Minneapolis, MN, USA). Olympus MVX10 was used for microscopic observation. Photographs from the slide sections were obtained by using Olympus BX51.

Statistics evaluation. Body weight, tumor volume, and tumor weight were evaluated by using SAS (Version 9.3, SAS Institute Inc., USA). Values are expressed as mean \pm SD. Bartlett test was used for homoscedasticity and Dunnett's $t$-test was used for statistical significance. Statistical significance was determined at $p<0.05$.

\section{Results}

SMT01, the cytotoxic NK cells expanded from PBMC. To produce SMT01, NK cells were isolated from human peripheral blood by using MACSexpress, depleting CD3-positive T cells. The isolated NK cells were then cultured for 14 days in the presence of IL2. To determine SMT01's cell population, the ex vivo expanded cells were stained with different antibodies detecting NK cell-antigens CD56/CD3, T cell-antigen CD3, and other lymphocyte antigens CD40 to identify DC and B cells. 

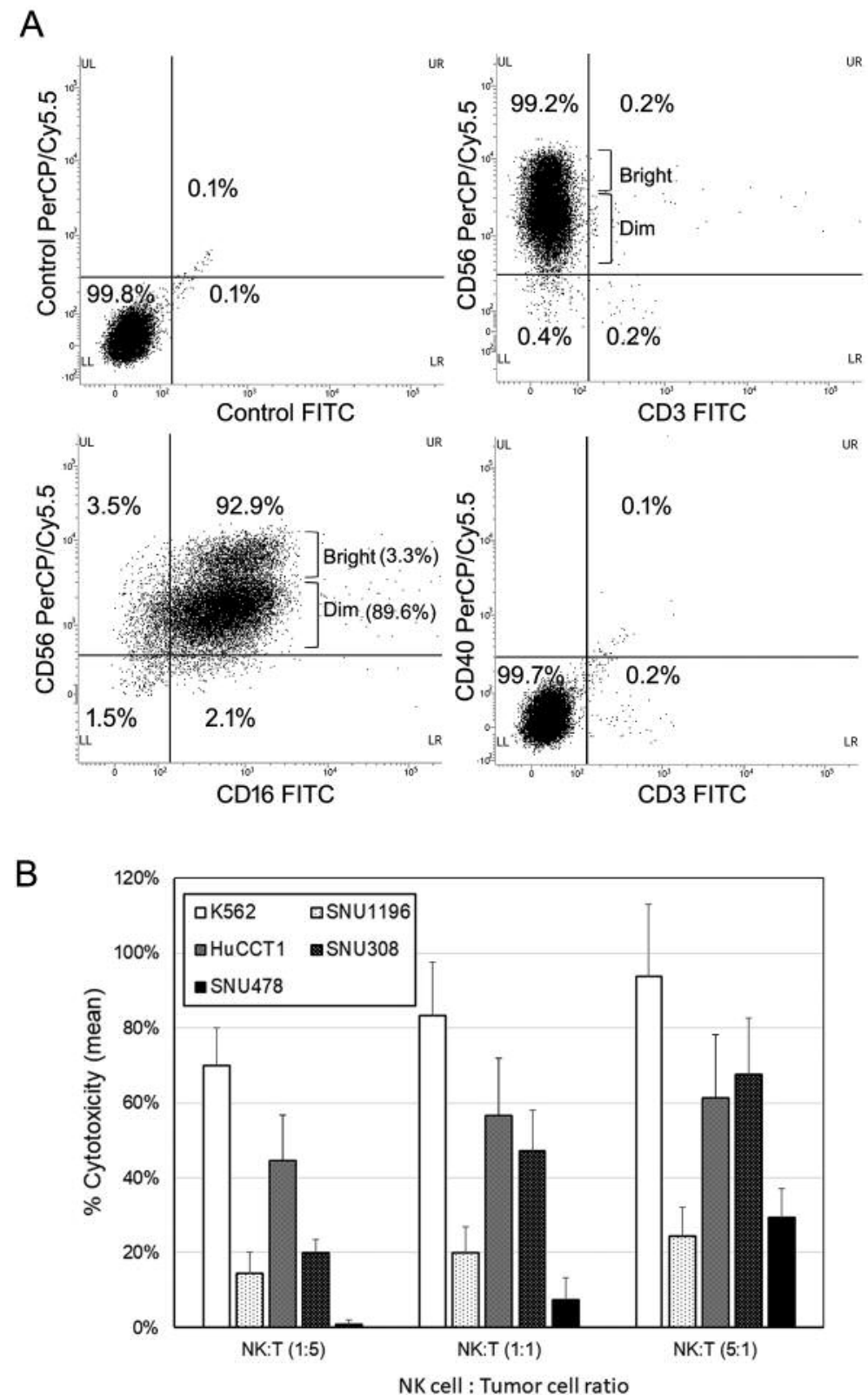

Figure 2. Flow cytometry of the ex vivo expanded SMT01 and cytotoxicity against cholangiocarcinoma cell lines. A: Phenotypic cell population of SMT01. Anti-CD56/CD16 antibodies were used to determine types of NK cells and anti-CD3 antibody was used to detect T cell population. AntiCD40 antibody recognizes DC and B cells. B: Cytolytic evaluation of SMTO1 against intrahepatic and extrahepatic CC cell lines: HuCCT-1 (intrahepatic), SNU1196 (extrahepatic), SNU308 (extrahepatic), and SNU478 (ampulla of Vater). K562 cell lines were used as a positive control.

CD56/CD16 antibodies were also used to determine the NK cells subtypes. Based on CD56 and CD3 phenotype, the majority of the expanded cells represented NK cells while $0.2 \%$ was $\mathrm{CD}^{+} \mathrm{T}$ cell population (Figure $2 \mathrm{~A}$ ). $99.2 \%$ of SMT01 cell population was defined as $\mathrm{CD}^{+} 6^{+} / \mathrm{CD}^{-} \mathrm{NK}$ cells. $\mathrm{CD}^{-\mathrm{dim}}$ and CD56 ${ }^{\text {bright }}$ types of NK cells were also detected, revealing that SMT01 contained mostly CD56 ${ }^{\mathrm{dim}}$ NK cells.
Cytolytic activity of SMT01 was also evaluated (Figure 2B). To determine cytotoxicity of NK cells, Cell Counting Kit-8 (CCK-8) was effectively used to count the number of viable target cells. K562 cells were included as a positive control to compare the viability of the human intrahepatic and extrahepatic cholangiocarcinoma (CC) cell lines. As expected, SMT01 showed an enhanced cytolytic activity to 
Table II. Summary of histopathological findings.

\begin{tabular}{|c|c|c|c|c|c|c|c|c|c|c|}
\hline \multirow{2}{*}{$\begin{array}{l}\text { Group/ } \\
\text { Dose }\end{array}$} & \multirow[t]{2}{*}{$\mathrm{N}$} & \multicolumn{4}{|c|}{ Apoptosis } & \multicolumn{5}{|c|}{ Necrosis } \\
\hline & & Rate $(\%)$ & \pm & + & ++ & \pm & + & ++ & +++ & ++++ \\
\hline G1/0 cells/head & 8 & $8.17 \pm 1.26$ & 8 & 0 & 0 & 0 & 5 & 3 & 0 & 0 \\
\hline $\mathrm{G} 2 / 4 \times 10^{4}$ cells $/$ head & 8 & $12.42 \pm 2.59$ & 2 & 6 & 0 & 0 & 1 & 5 & 1 & 1 \\
\hline $\mathrm{G} 3 / 2 \times 10^{5}$ cells $/$ head & 8 & $11.71 \pm 3.73$ & 3 & 5 & 0 & 1 & 3 & 2 & 2 & 0 \\
\hline $\mathrm{G} 4 / 1 \times 10^{6}$ cells $/$ head & 8 & $10.88 \pm 2.25$ & 3 & 5 & 0 & 0 & 4 & 3 & 1 & 0 \\
\hline $\mathrm{G} 5 / 3+120 \mathrm{mg} / \mathrm{kg}$ & 8 & $23.00 \pm 3.35$ & 0 & 2 & 6 & 0 & 4 & 2 & 2 & 0 \\
\hline
\end{tabular}

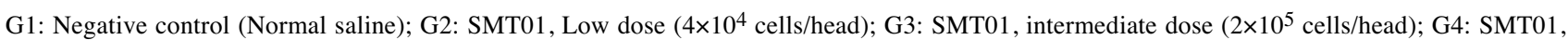
High dose $\left(1 \times 10^{6}\right.$ cells/head); G5: Cis diammineplatinum (II) dichloride (CDDP)+Gemcitabine (Gem); N: number of animals; Grade- \pm : minimal, +: mild, ++: moderate, +++ : marked, ++++ : severe.

K562 cells. Among the CC cell lines, SMT01 showed the highest cytolytic activity to HuCCT1 and SNU308 relatively to SNU1196 and SNU478 cells. This suggested that NK cells displayed variable cytolytic effects depending on the various cancer types.

In vivo study of SMT01 in nude mice. In vivo study was done to evaluate SMT01 in two different aspects, dosage dependent safety and toxicity and dosage efficacy at three different concentrations of the NK cells (Tables I and II). Intravenous infusion of SMT01 was administrated by injecting the cells through tail venous of nude mice. Low dose of SMT01 was $4 \times 10^{4}$ cell/animal which corresponds to $2 \times 10^{6}$ cells $/ \mathrm{kg}$ in human. $2 \times 10^{5}$ cells/animal and $1 \times 10^{6}$ cells/animal were the intermediate and high doses of the infusion, respectively. For the dosage dependent safety and toxicity study, a total of 18 SMT01 infusions (2 injections per 3 weeks) were performed at the three different dose levels (Table I, Figure 1). The result showed that the mice tolerated well the maximum dose of SMT01 and no death caused from SMT01 infusion. All of the treatment groups did not show toxicity response related to the $\mathrm{NK}$ cell administration in the internal organs (data now shown). No significant change of $\mathrm{T}$ and $\mathrm{B}$ lymphocytes was observed, suggesting that SMT01 did not cause immune-related adverse events (Table I).

In vivo cytolytic activity of SMT01 against $\mathrm{CC}$ was evaluated. To do this, xenograftment of $\mathrm{CC}$ cells into nude mice was performed. HuCCT- 1 cells at $5 \times 10^{6}$ cells $/ \mathrm{ml}$ were subcutaneously transplanted on the back of 10 nude mice in each group. After 8 days of CC cell transplantation, well engrafted with similar tumor volumes nude mice, were carefully selected and used for the in vivo study. For treatment, eight selected mice from each treatment group including negative ( $\mathrm{G} 1$, normal saline infusion) control group were intravenously administrated (Table II, Figure 1). Chemo-administration as a positive control group (G5,
$\mathrm{CDDP}+\mathrm{Gem}$ ) was also done with the same number of mice. After five administrations of the SMT01 divided into 3 different dose concentrations (G2-G4), the tumors were dissected out from the nude mice and the tumor volume and weight were measured (Figure 3 ).

The mean tumor volumes observed at 55 days after administration were 5324, 2769, 3491, 3193, and $2167 \mathrm{~mm} 3$ for G1, G2, G3, G4, and G5, respectively (Figure 3B). All of the treatment groups except the negative control group (G1) showed tumor growth inhibition. Extracted mean tumor weights from the groups also revealed significant differences between the treatment groups and the negative control group (Figure 3A). The results indicated that SMT01 administration showed a significant effect on tumor growth inhibition. Although maximum growth inhibition was observed at the chemo-administration group, no statistical significance was obtained. More interestingly, however, when body weight changes of all the treatment groups were evaluated, the chemo-administrated group of mice showed significantly lowered body weight (Figure 4A) and this was not related to a food consumption problem (Figure 4B). All of the other treatment groups of mice showed similar weight maintenance. The results indicate that NK cell therapy has a beneficial effect on weight maintenance which is relevant to the quality of life, an important parameter in cancer therapeutic.

Taken together, the in vivo study revealed that SMT01 apparently displayed cytolytic activity against cholangiocarcinoma without significant side effects related to cell infusions.

Histologic evaluation of SMTO1 activity in nude mice tumor. SMT01's cytolytic activity was evaluated by using tumor tissue sections (Figures 5 and 6). Anti-human CD56 antibody was efficiently used for IHC experiment to detect penetration of NK cells into the HuCCT-1 tumor of nude mice administrated with SMT01 infusion. The same tumor tissue sections were used for TUNEL assay. 

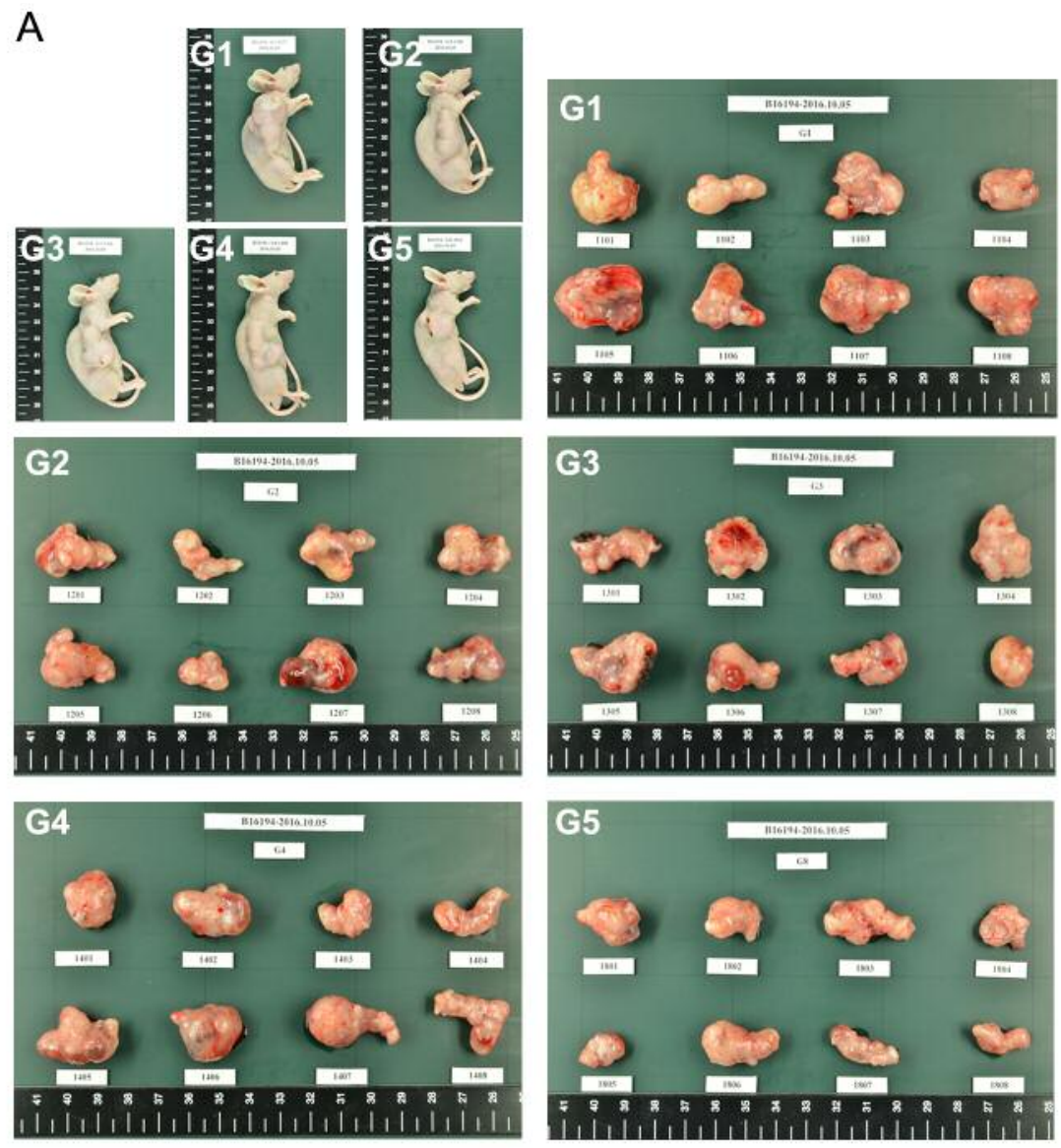

B

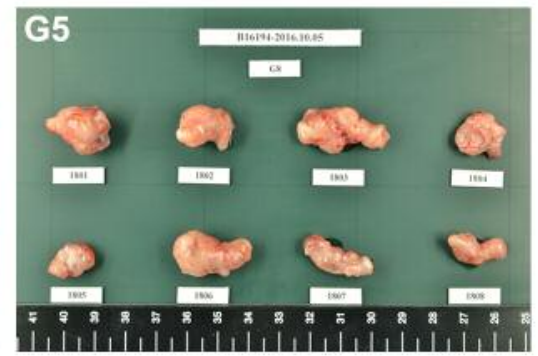

C
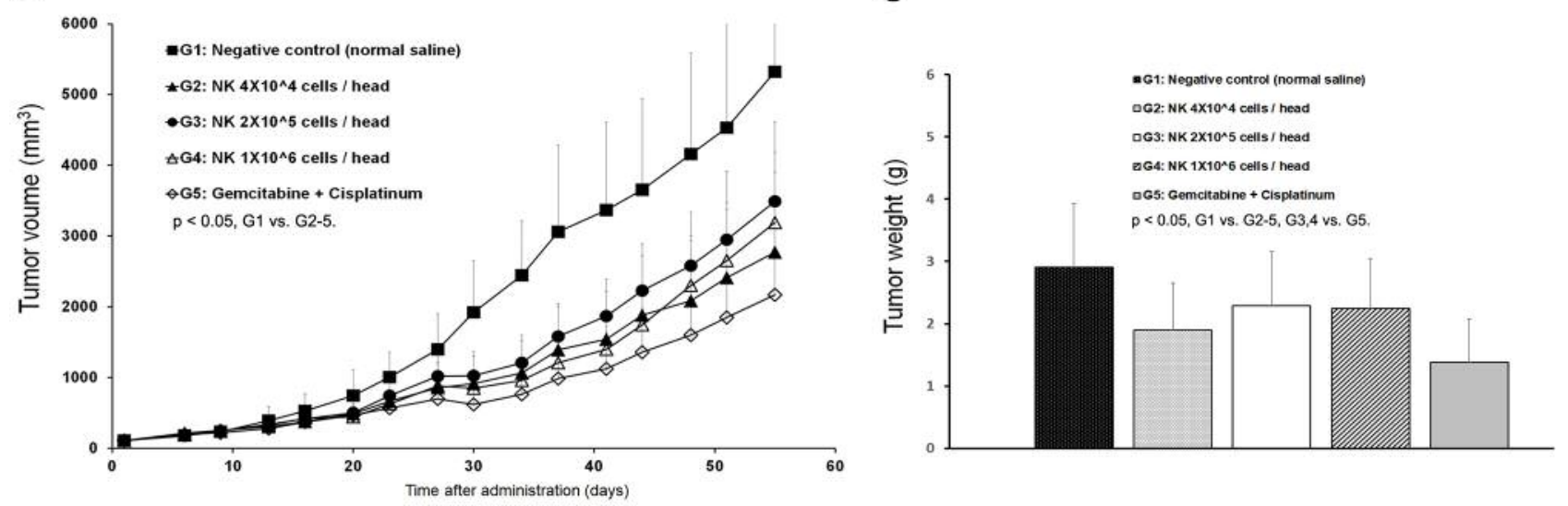

Figure 3. Tumor growth inhibition of SMT01. A: Five different groups of nude mice and the tumors dissected out from the mice. G1, Normal saline;

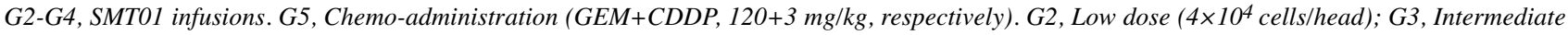
dose $\left(2 \times 10^{5}\right.$ cells/head); G4, High dose $\left(1 \times 10^{6}\right.$ cells/head $)$. B: Tumor volume. C: Tumor weight.

IHC of tissue sections from G1 (normal saline infusion mice) and G5 (Chemo-administrated mice) groups of mice showed CD56 positive signal at background level. IHC of tissue sections from the SMT01 infusion groups (G2-G4) clearly showed CD56-positive cells mainly located at the cleavage between the tumor nodules, suggesting that SMT01 penetrated into the HuCCT-1 tumor developed in the nude mice (Figure 5). The IHC signals were found to be located where the tissue 


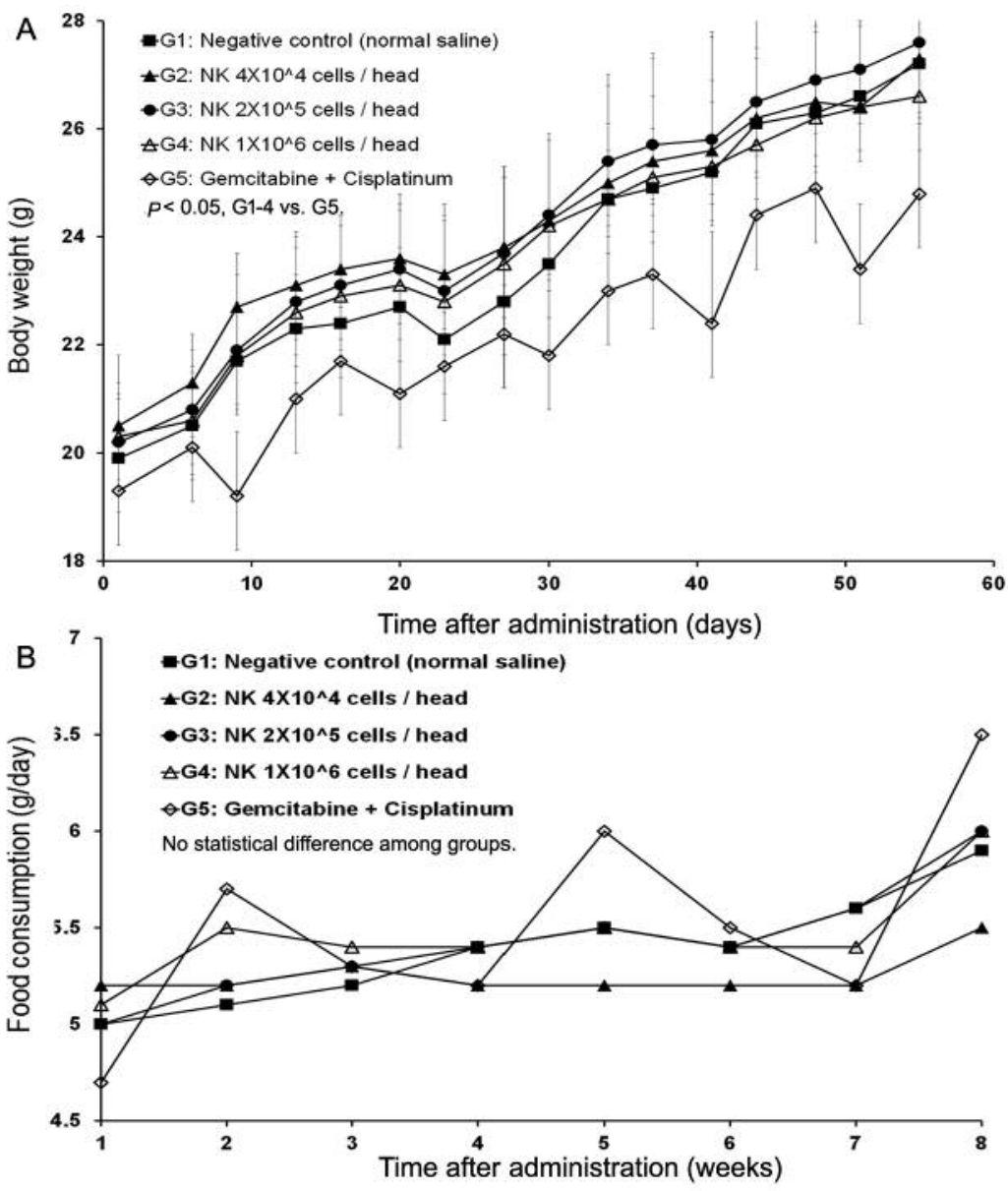

Figure 4. In vivo nude mice study of SMT01. A: Body weight change. B: Tumor volume change. Tumor volume was measured by using a caliper $\left(C D-15 C X\right.$, Mitutoyo, Japan) and calculated as: TV $\left(\mathrm{mm}^{3}\right)=L(\mathrm{~mm}) \times W^{2}\left(\mathrm{~mm}^{2}\right) \times 1 / 2$. G2 (Low dose) showed statistical significance p $<0.01$ at days 23, 27, 30, 34, 44, 48, 51 and 55 during administration ( $p<0.05$ at day 37, 41). G3 (Intermediate dose) $p<0.05$ at day 30, 37, 41, 51, and 55 (day 34, 44, and $48 p<0.01$ ). G4 (High dose) $p<0.01$ at day 23, 30, 34, 37, 41, 44, 48, 51, and 55 ( $p<0.05$ at day 20 and 27). G5 (CDDP+Gem) $p<0.01$ at day 23-55). C: Tumor weight. Measured mean tumor weights: G1, $2.904 \mathrm{~g}$ (IR=0.0\%); G2, $1.892 \mathrm{~g}(\mathrm{IR}=34.8 \%) ; \mathrm{G3}, 2.292 \mathrm{~g}(\mathrm{IR}: 21.1 \%)$; G4, $2.252 \mathrm{~g}(\mathrm{IR}=22.4 \%) ; \mathrm{G5}, 1.385 \mathrm{~g}(\mathrm{IR}: 52.3 \%)$. IR $(\%)=(1-\mathrm{T} / \mathrm{C}) \times 100$ (T: Mean tumor weight of the test substance group and positive substance group C: Mean tumor weight of the negative control group).

mass and density were loose inside the tumor due to feeding vessel penetration (Figure 5, G2-G4, arrowhead).

Infiltration of CD56-positive NK cells of the tumor tissue was clearer at higher resolution of microscopy (Figure 6B). As shown in Figure 6B, the CD56-positive NK cells were densely detected at sites close to blood vessels in the mouse groups (G2-G4) received SMT01 infusion. Similar result was also obtained by IHC against a NK cell activating receptor, NCR1, confirming that the penetrating cells were NK cells (Figure 6B, see NCR1 panel figures). Apoptosis was also detected by TUNEL staining and IHC against the apoptotic marker caspase 3a (Figure 6A and B). The results revealed that apoptotic tumor cells largely appeared in the tumor of SMT01 infused and chemo-administration mice groups
(Figure 6A). Interestingly, apoptotic cells were more frequently detected at the vicinity of the populated NK cells. The result suggested that the extravasated NK cells exerted their cytolytic activity primarily against tumor cells near the tumor vessels. Taken together, the results revealed that SMT01 displays cytotoxicity against cholangiocarcinoma.

\section{Discussion}

Cytolytic activity of NK cells (SMTO1) against CC cells. Cholangiocarcinoma (CC) is a malignant tumor that originates from the biliary duct epithelium. The carcinoma is largely classified into two types: intrahepatic and extrahepatic CC (19). Diagnosis and curative treatment for 


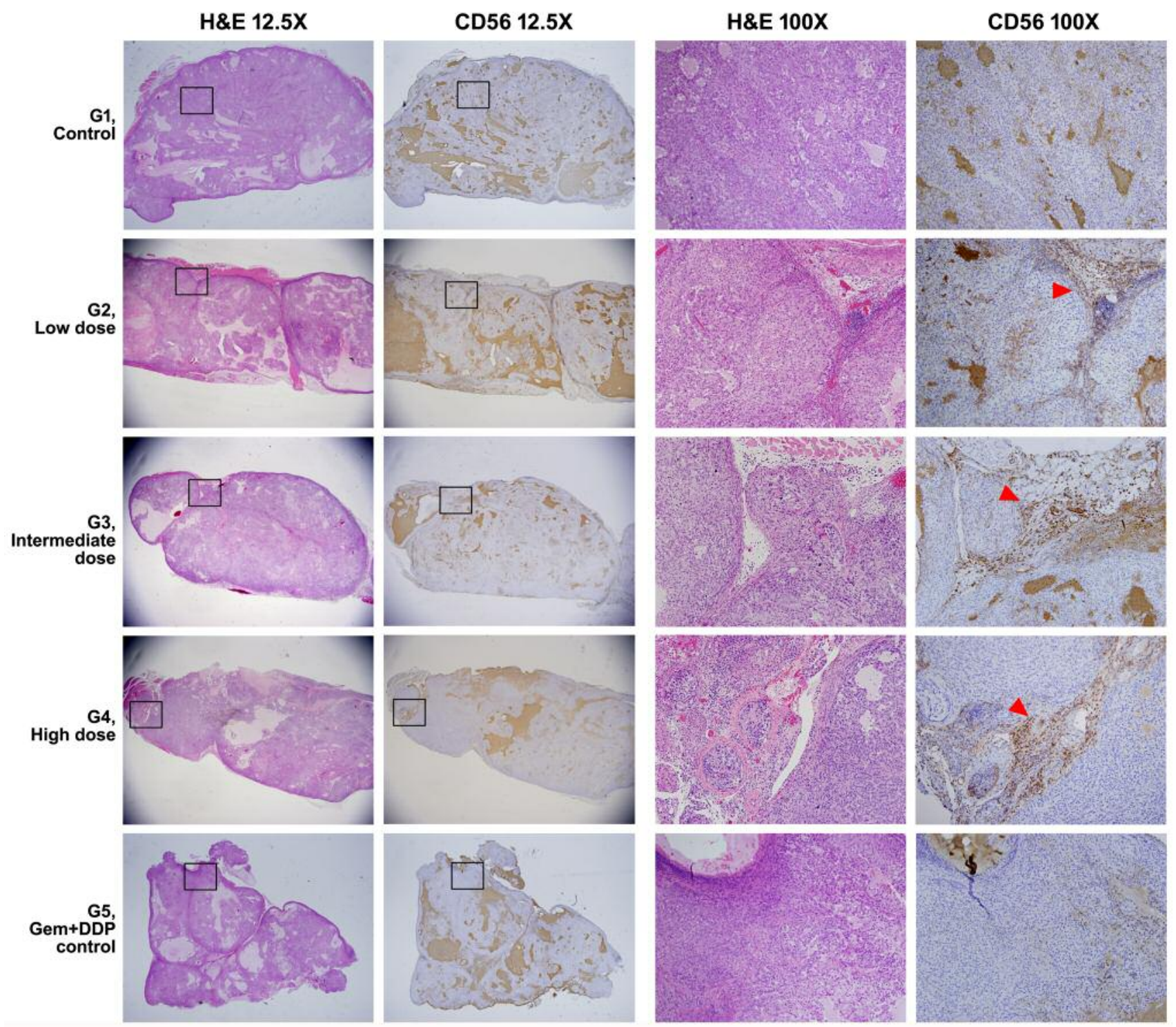

Figure 5. H\&E stain and IHC experiment of the nude mice tumor. Full morphology and CD56 IHC finding of the representative tumors from each group is shown at a low magnification (12.5X). Magnified views (H\&E and IHC images from consecutive sections) of rectangular boxes are shown on the right columns (100X). CD56-positive NK cells in the group 2-4 (G2-G4) are mainly located along the cleavage between tumor nodules (red arrowheads).

$\mathrm{CC}$ are not easy because pathogenesis of the malignancy is not fully understood while the disease is more likely aggressive, infiltrating surrounding tissues and thus most of the patients are inoperable at the time of diagnosis $(20,21)$. Also, an alternative regimen for replacing the standard chemotherapy is not available when the chemotherapy fails to give a clinical response. Currently, Gem/CDDP regimen is widely administered to patients at the inoperable stage. The regimen however decreases quality of life (QOL) due to toxicity.
Our interest in developing an immune cell therapy for CC is to provide an alternative treatment for the patients diagnosed with an inoperable stage or who have had chemofailure as a 2nd line therapy. NK cells are one of the lymphocytes with natural cytotoxicity to malignant cells. For this reason, killing effect of the immune cells against various types of cancer have been intensively studied in vitro using the cancer cells (22) and also in vivo using animal models (23). NK cells are now widely used as an innovative immune-therapy. While autologous NK cell therapy has 


\section{A TUNEL stain}
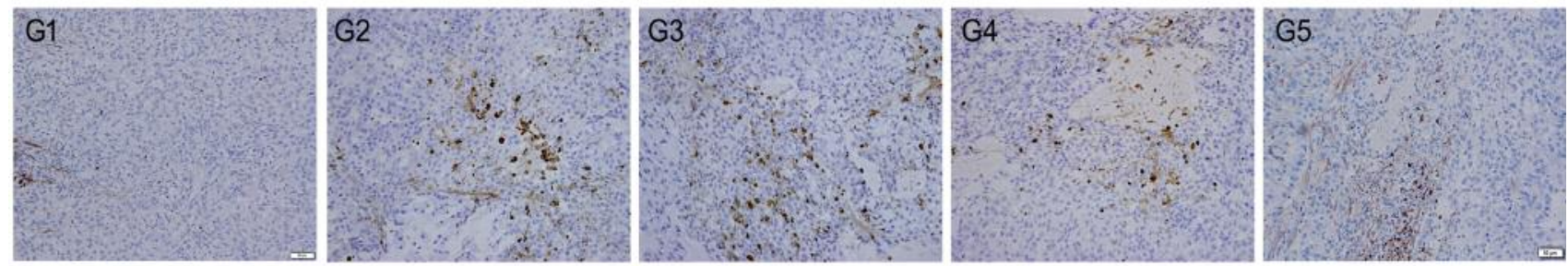

\section{B Immunohistochemical stain}
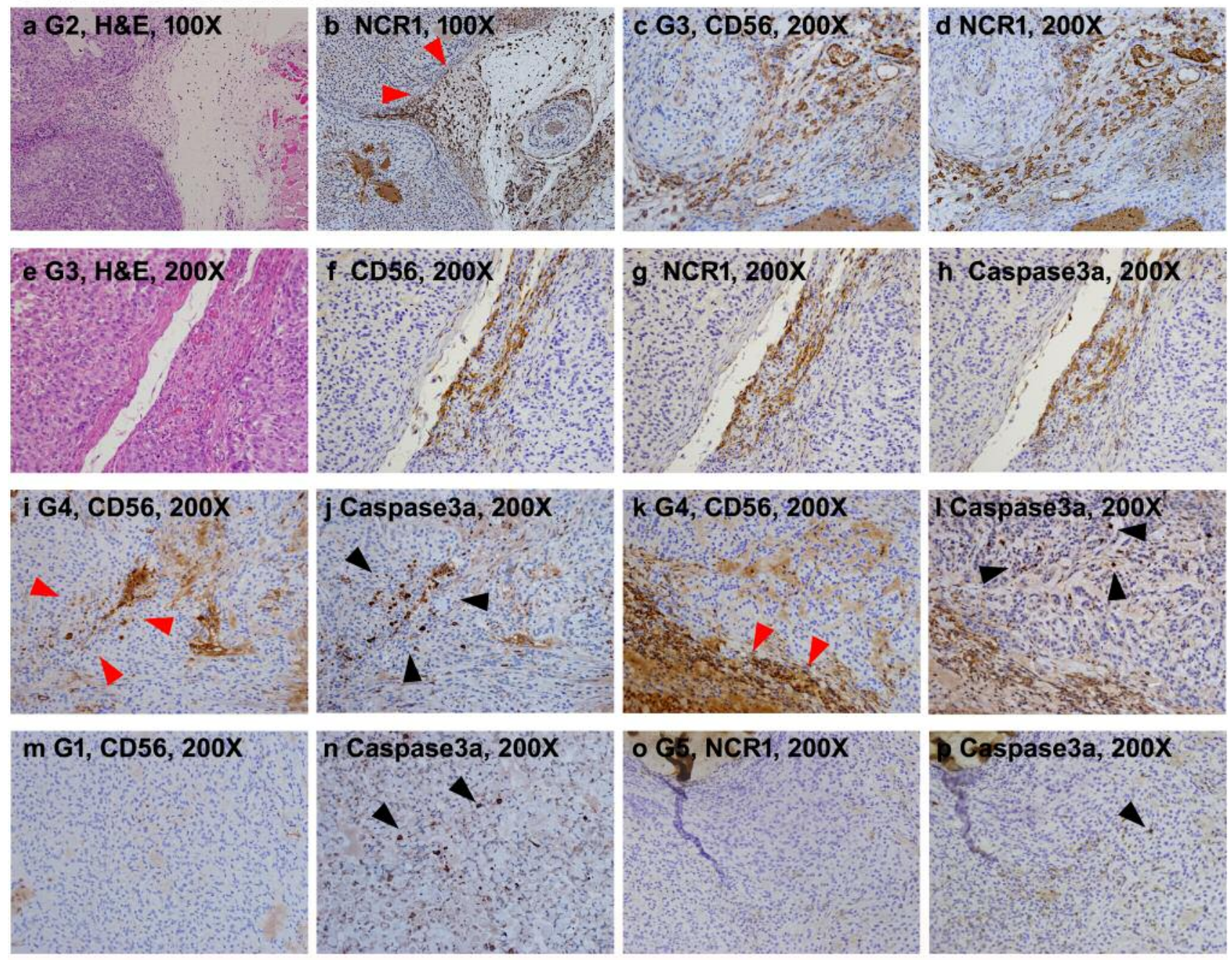

Figure 6. Histological observation of apoptotic tumor cells and infiltrating NK cells. A: TUNEL stains of all study group's tumor sections. TUNELpositive cells were significantly more frequent in G2-G5 compared to G1. B: IHC experiments. a and b: Consecutive section images from G2 showing NCR1-positive NK cell infiltration (red arrowheads) along the loose tissue between tumor nodules. $c$ and d: Consecutive section images from G3 showing precisely matched CD56 and NCR1-positive NK cells. e-h: Consecutive section images from G3. Apoptotic signals of the tumor cells detected by IHC with anti-human caspase 3 a antibody are predominantly detected at the area with robust NK cell infiltration. $i$ and $j$ : Consecutive section images from G4. In the tumor, caspase3a-positive cells (black arrowheads) are detected at the vicinity where infiltrated NK cells are detected (red arrowheads). $k$ and $l$ : Consecutive section images from G4. Caspase3a-positive cells (black arrowheads) are located at the vicinity of NK cell infiltration (red arrowheads) along loose tissue between tumor nodules. m-p: Consecutive section images from G1 and G5 showing spotty caspase3apositive cells (black arrowheads) in the tumor irrelevant to NK cell infiltration. 
previously shown limited clinical response, many of the molecular and clinical studies have now focus on the use of NK cells prepared from healthy blood donors overcoming the clinical weaknesses of using autologous NK cells. Unfortunately, however, no NK cell therapy for CC has been available. Thus, the primary goal of this in vivo study by using a nude mouse model was to determine whether NK cell therapy is clinically potent $\mathrm{CC}$.

In this study, ex vivo expanded NK cells (SMT01) were used to investigate the cytolytic activity of cells against $\mathrm{CC}$. As demonstrated in Figure 2A, SMT01 are highly purified NK cells with T cells depletion. These NK cells were used in in vitro and in vivo studies to examine their cytotoxicity activity against CC cells. In-vitro study of SMT01 was efficiently performed by the CCK-8 assay in which the number of target cells survived following administration of NK cells was measured and the results showed that SMT01 displayed an enhanced cytolytic activity to HuCCT-1 and SNU308 cells and somewhat lower activity to SNU1196 and SNU479 cells (Figure 2B). Further in vivo analysis of the cytolytic activity of SMT01 was performed by choosing HuCCT-1 cells.

In vivo study of SMT01 and pre-clinical relevance of NK cell therapy against CC. In vivo cytolytic activity of SMT01 was investigated by generating a xenografted $\mathrm{CC}$ tumor in nude mice. To do this, we selected and transplanted HuCCT-1 cells into nude mice. The xenografted nude mice, grouped into 3 different dosage groups, received multiple infusions of SMT01 (Figure 1 and Table II). Normal saline infusion was used as a negative control group $(\mathrm{G} 1)$ and chemo-administration with CDDP+Gem (G5) was used as a positive control. The cytolytic activity in these groups was compared to that of the SMT01 infusion groups (G2-G4). When a total of five treatments was given to the different nude mice groups, SMT01 infusions were found to inhibit $\mathrm{CC}$ tumor growth (Figure 3A). Measurement of tumor volume and weight done by dissecting out the $\mathrm{CC}$ tumors from the xenografted nude mice revealed that the negative control group (G1) administrated with normal saline contained the largest tumor volume and also the heaviest weight, as expected, while all of the other treatment groups showed a significantly reduced tumor volume and weight, suggesting that both NK cell therapy and chemo-therapy effectively worked to kill CC cells (Figure 3B). When the body weight of the nude mice was measured, however, that of the chemo-administrated group (G5) was found to be maintained at the lowest level among the five groups (Figure 4A) and this was not related to food consumption (Figure 4B). This suggests that chemo-administration is more stressful and is not beneficial in relevance to QOL, although the treatment showed the highest inhibitory effect on CC tumor growth.

As the dosage dependent safety and toxicity concerns that may be caused from SMT01 infusions, a total of 18 repeated SMT01 infusions were performed by intravenous tail injection to the nude mice with 10-day intervals for a total of 27 weeks (Figure 1A). The nude mice well tolerated all three doses, low (G2), intermediate (G3), and high (G4) (Table I). No death or $\mathrm{SAE}$ related to NK cell infusion was observed. The result suggested that SMT01 administration is at least pre-clinically safe. Unexpectedly, dose-dependent tumoricidal effect of NK cells, was not observed.

Histological evidence of SMT01 invasion into CC tumor. Histology, IHC, and TUNEL experiments were performed to demonstrate SMT01 activity inside the HuCCT-1 tumor (Figures 5 and 6). To do this, the tumors dissected out from the nude mice were used to prepare the consecutive tissue section for those experiments. Despite the fact that histologic evaluation was performed 14 days after the last infusion of SMT01 (Figure 1B), CD56-positive NK cells infiltrated into the tumors of all SMT01 infusion groups were observed (Figures 5 and 6). Considering lifespan of NK cells to be approximately 2 weeks (24), it was quite interesting to observe a robust NK cell infiltration into the tumors (Figures 5 and 6B, red arrowheads). This may indicate the xenogenic nature of SMT01 treatment with a prolonged cytolytic activity against $\mathrm{CC}$ cells in mice.

TUNEL staining (Figure 6A) and IHC experiment for caspase 3a (Figure 6B) also suggested that apoptosis was underway inside the tumor. Unlikely the immune cell therapy application for hematologic malignancies, solid cancers generally do not show penetration of the immune cells deep into the tumor mass. We also found infiltrating CD56positive NK cells densely and primarily located along the tumor vessels, and the apoptotic tumor cells were found at the vicinity of the populated NK cells. These findings suggest that the dying tumor cells were caused truly by the extravasated NK cell's. In deep sites of tumor mass, however, NK cells were seldomly observed, indicating that immune cell penetration remains an unsolved issue. The histopathological findings of the tumors from the five different groups are summarized in Table II.

In summary, this in vitro and in vivo study with $\mathrm{NK}$ cells demonstrated their cytotoxic effect to $\mathrm{CC}$ cells. The preclinical study results obtained from nude mice bearing $\mathrm{CC}$ tumors may not be the same or similar when the NK cell therapy is clinically applied to CC patients. Thus, further clinical study with SMT01 against CC is necessary to investigate clinical responses.

\section{Conflicts of Interest}

The Authors have no conflicts of interest to disclose.

\section{Acknowledgements}

The Authors thank all blood donors for consent and participation. The Authors also thank all staffs involved in the SMT01 study. 


\section{References}

1 Global Burden of Disease Cancer Collaboration: The global burden of cancer 2013. JAMA Oncol 1: 505-527, 2015.

2 Bannales JM, Cardinale V, Carpino G, Marzioni M, Andersen JB, Invernizzi P, Lind GE, Folseraas T, Forbes SJ, Fouassier L, Geier A, Calvisi DF, Mertens JC, Trauner M, Benedetti A, Maroni L, Vaquero J, Macias RI, Raggi C, Perugorria MJ, Gaudio E, Boberg KM, Marin JJ and Alvaro D: Cholangiocarcinoma: current knowledge and future perspectives consensus statement from the European Network for the Study of Cholangiocarcinoma (ENS-CCA). Nature Rev 13: 261-280, 2016.

3 Ghouri YA, Mian I and Blecharcz B: Cancer review: Cholangiocarcinoma. J Carcinog 14: 1, 2015.

4 Kay HD, Fagnani R and Bonnard GD: Cytotoxicity against the K562 erythroleukemia cell line by human natural killer (NK) cells which do not bear free Fc receptors for IgG. Int J Cancer 24: 141-150, 1979.

5 Stern P, Gidlund M, Orn A and Wigzell H: Natural killer cells mediate lysis of embryonal carcinoma cells lacking MHC. Nature 285: 341-342, 1980.

6 Purdy AK and Campbell KS: Natural killer cells and cancer: regulation by the killer cell Ig-like receptors (KIR). Cancer Biol Ther 8: 2211-2220, 2009.

7 Lanier LL: NK cell recognition. Annu Rev Immunol 23: 225274, 2005

8 Diefenbach A, Jensen ER, Jamieson AM and Raulet DH: Rae1 and H60 ligands of the NKG2D receptor stimulate tumour immunity. Nature 413: 165-171, 2001.

9 Smyth MJ: NKG2D function protects the host from tumor initiation. J Exp Med 202: 583-588, 2005.

10 Robertson MJ and Ritz J: Biology and clinical relevance of human natural killer cells. Blood 76: 2421-2438, 1990.

11 Lanier LL, Testi R, Bindl J and Phillips JH: Identity of Leu-19 (CD56) leukocyte differentiation antigen and neural cell adhesion molecule. J Exp Med 169: 2233-2238, 1989.

12 Cooper MA, Fehniger TA and Caligiuri MA: The biology of human natural killer-cell subsets. Trends Immunol 22: 633-640, 2001 .

13 Ruggeri L, Capanni M, Urbani E, Perruccio K, Shlomchik WD, Tosti A, Posati S, Rogaia D, Frassoni F, Aversa F, Martelli MF and Velardi A: Effectiveness of donor natural killer cell alloreactivity in mismatched hematopoietic transplants. Science 295: 2097-2100, 2002.
14 Ruggeri L, Mancusi A, Perruccio K, Burchielli E, Martelli MF and Verlardi A: Natural killer cell alloreactivity for leukemia therapy. J Immunother 28: 175-182, 2005.

15 Cheng M, Chen Y, Xiao W, Sun R and Tian Z: NK cell-based immunotherapy for malignant diseases. Cell Mol Immunol 10: 230-252, 2013.

16 Petrie-Hanson L, Hohn C and Hanson L: Characterization of rag1 mutant zebrafish leukocytes. BMC Immunol 10: 1-8, 2009.

17 Jung IH, Jung DE, Park YN, Song SY and Park SW: Aberrant Hedgehog ligand induce progressive pancreatic fibrosis by paracrine activation of myofibroblasts and ductular cells in transgenic zebrafish. PLoS One 6: 1-15, 2011.

18 Park SW, Davison JM, Rhee J, Hruban RH, Maitra A and Leach SD: Oncogenic KRAS induces progenitor cell expansion and malignant transformation in zebrafish exocrine pancreas. Gastroenterology 134: 2080-2090, 2008.

19 Lazaridis KN and Gores GJ: Cholangiocarcinoma. Gastroenterology 128(6): 1655-1667, 2005.

20 Patel T: Worldwide trends in mortality from biliary tract malignancies. BMC Cancer 2: 10, 2002.

21 Patel T: Increasing incidence and mortality of primary intrahepatic cholangiocarcinoma in the United States. Hepatology 33(6): 1353-1357, 2001.

22 Shin S, Kim M, Lee SJ, Park KS and Lee CH: Trichostatin A Sensitizes Hepatocellular Carcinoma Cells to Enhanced NK Cell-mediated Killing by Regulating Immune-related Genes. Cancer Genomics Proteomics 14: 349-362, 2017.

23 Konagai A, Yoshimura K, Hazama S, Yamamoto N, Aoki K, Ueno T, Fujioka M, Iijima H, Kato M, Uchida M, Wada T, Inoue M, Asao T, Fuse M, Wada S, Kuramasu A, Kamei R, Takeda S, Yamamoto S, Yoshino S, Oka M and Nagano H: Correlation between NKG2DL expression and antitumor effect of proteinbound polysaccharide- $\mathrm{K}$ in tumor-bearing mouse models. Anticancer Res 37(8): 4093-4101, 2017.

24 Hochman PS, Cudkowicz G and Dausset J: Decline of natural killer cell activity in sublethally irradiated mice. J Natl Cancer Inst 61: 265-268, 1978.

Received March 16, 2018

Revised April 27, 2018

Accepted May 2, 2018 